\title{
Gab3 is required for human colorectal cancer cell proliferation
}

XIANG Shihao co*, WANG Na co, HUI Pingping and MA Jiali

\begin{abstract}
Here, we focused on the potential function of Gab3, an uncommon Gab family protein, in human colorectal cancer (CRC) cells. We found that Gab3 was only expressed in human colon cancer tissues as well as in established (HCT-116 and HT-29 lines) and primary human CRC cells. It was however absent in normal human colon cancer tissues and in FHC colon epithelial cells. Knockdown of Gab3 by targeted-shRNAs inhibited proliferation of the CRC cells. Reversely, exogenous overexpression of Gab3 promoted CRC cell proliferation. At the signaling level, Gab3 co-precipitated with p85 and SHP2 in CRC cells, which was required for subsequent Akt and Erk activation. Gab3 shRNA knockdown inhibited Akt and Erk activation, yet Gab3 over-expression augmented it. In vivo, HCT-116 xenograft tumor growth in severe combined immune deficient (SCID) mice was suppressed following expressing Gab3 shRNAs. Meanwhile, Akt and Erk activation in Gab3 shRNA-expressing tumors was also largely inhibited. Together, our results suggest that Gab3 expression in CRC cells is important for Akt-Erk activation and cell proliferation.
\end{abstract}

Keywords. Colorectal cancer; Gab3; Akt; Erk; Oncogene; 


\section{Introduction}

Colorectal cancer (CRC) causes large mortalities around the world each year [1,2,3]. Although significant improvements have been achieved for the treatment of CRC, the overall survival (OS) has not been improved for the advanced, metastatic or recurrent CRC [1,2,3,4]. Meanwhile, in China [5,6] and other regions, CRC's incidence has been rising [7,8]. Existing studies have suggested that CRC's molecular heterogeneity obstructs the clinical application of specific molecularly-targeted agents $[1,2,3]$. Therefore, recent researches focused on identifying novel oncogenic protein (or oncogenes) of CRC $[1,2,3,7,9]$.

Gab3 is the least common member of Gab [growth factor receptor binding 2 (Grb2)-associated binding protein] family adaptor proteins, which also include two other well-known members: Gab1 and Gab2 [10,11]. Gab3 expression is more restricted to the hematopoietic tissues, including spleen and thymus $[12,13,14]$. Yet, low expression of Gab3 is also detected in other tissues (i.e. heart, kidney, uterus, and brain) $[12,13,14]$. As an adaptor protein, Gab3 could be tyrosine phosphorylated, which then associates with the $\mathrm{SH} 2$ domain-containing proteins, i.e. PI3K regulatory subunit p85 and src homology-containing tyrosine phosphatase 2 (SHP2) $[12,13,14]$. This presumably will mediate activation of Akt and Erk signaling cascades $[12,13,14]$. The current research studied expression and the potential function of Gab3 in human CRC cells.

\section{Materials and methods}

2.1. Reagents. All the antibodies of this study were purchased from Santa Cruz Biotech (Shanghai, China) and Cell Signaling Tech (Shanghai, China). The reagents for cell cultures were obtained from Gibco (Shanghai, China).

2.2. Cell cultures. The established human CRC cell lines, HT-29 and HCT-116, as well as one line of primary human colon cancer cells $[15,16]$ were provided by Dr. Lu at Nanjing Medical University (Nanjing, China). The cultures of these cells were described in detail previously [15,16]. FHC normal colon epithelial cell line was obtained from iBS Fudan Cell Bank (Shanghai, China). FHC cells were cultivated in DMEM plus FBS (10\%) medium. To count cells, the number of viable cells (trypan blue negative) was recorded via a TC20 automatic counter (Bio-Rad, Shanghai, China).

2.3. Human tissue specimens. Human tissue specimens (stored in liquid nitrogen) were provided by Dr. Lu's group at Nanjing Medical University $[16,17,18,19]$. The surgery-isolated colon cancer 
specimens $(n=10)$ and paired surrounding normal colon tissues $(n=10)$ were thoroughly washed and minced into small pieces. Samples were then mechanically dissociated. Tissues were then lysed by the tissue lysis buffer (Sigma). Expressions of Gab3 and $\beta$-actin in tissue lysates were tested. The study was conducted according to the principles expressed in the Declaration of Helsinki. The protocol was approved by Shanghai Jiao Tong University School of Medicine. Written-informed consent was obtained from each participant.

2.4. Western blot assay. Following the applied treatment/s, $30 \mu \mathrm{g}$ protein lysates per sample were separated by SDS-PAGE gel (10-12\%). Proteins were then transferred to PVDF membranes (BioRad, Shanghai, China). After blocking in $10 \%$ milk for $30 \mathrm{~min}$, the blots were incubated with designated primary and secondary antibodies. Detection of interested bands were through West Pico ECL reagents (Pierce, Shanghai, China). ImageJ software was applied to quantify the bands.

2.5. Co-immunoprecipitation (Co-IP). The detailed protocol of Co-IP assay was described early [20,21]. Briefly, cell lysates $(1000 \mu \mathrm{g}$ per treatment) were pre-cleared, which were then incubated with $0.05 \mu \mathrm{g}$ (per sample) of anti-Gab3 antibody (Santa Cruz) overnight at $4{ }^{\circ} \mathrm{C}$. The protein A/G PLUSagarose ( $40 \mu \mathrm{L} / \mathrm{sample}$ ) was then added to the supernatant for another 4 hours. Pellets were washed six times with PBS, resuspended in lysis buffer, and then assayed by Western blot to test SHP2 and p85 association.

2.6. Real-time quantitative polymerase chain reaction (RT-qPCR) assay. RNA in cellular samples was extracted by the SV reagents (Promega, Shanghai, China) and was reverse-transcribed. cDNA was amplified by RT-qPCR assay using a SYBR Green PCR kit (Applied Biosystems, Shanghai, China). The ABI 7600 System (Applied Biosystems, Shanghai, China) was utilized for the PCR reaction. Expression of Gab3 mRNA was tested using the ${ }^{\Delta \Delta} \mathrm{Ct}$ method, and $\beta$-actin was also examined as the internal control. The following primers were applied: Gab3, 5'GAGAGCCTCTCTTACACG-3' (sense) and 5'-GGCTGAAGCTGTGGGGTA-3' (antisense) [22]; $\beta$ actin: $\quad 5^{\prime}-T G A C G T G G A C A T C C G C A A A G-3^{\prime} \quad$ (sense) and 5' CTGGAAGGTGGACAGCGAGG-3' (antisense) [23,24].

2.7. BrdU incorporation assay of cell proliferation. As described [15], 5-bromo-2'deoxyuridine $(\mathrm{BrdU})$ incorporation was tested to reflect cell proliferation. In brief, CRC cells $\left(1 \times 10^{4 /}\right.$ well) with applied genetic modification were incubated with $\operatorname{BrdU}(10 \mu \mathrm{M}$, Roche, Shanghai, China) for 12 hours. Afterwards, cells were fixed, and BrdU incorporation was tested in an enzyme-linked 
immunosorbent assay (ELISA, Roche) format.

2.8. Clonogenic assay. CRC cells with applied genetic modification were seeded onto $10-\mathrm{cm}$ dish at 2000 cells/dish. After 10 days incubation, cells were fixed with $80 \%$ methanol and were then stained with $0.2 \%$ crystal violet. The number of colonies was counted manually.

2.9. shRNA stable knockdown of Gab3. A set of nine distinct Gab3 shRNAs were designed by Jikai Gene (Shanghai, China). These non-overlapping shRNAs were packed into the lentiviral GV428 vector, and were added to CRC cells for 24 hours. Afterwards, puromycin $(2.5 \mu \mathrm{g} / \mathrm{mL}$, Sigma) was utilized to select resistant colonies for 6-7 passages. Expression of Gab3 in the stable cells was tested by RT-qPCR assay and/or Western blot assay. Of the tested Gab3 shRNAs, two of them efficiently knocked down Gab3. They were named as "shGab3-\#1" and "shGab3-\#2". The scramble lentiviral shRNA ("sh-SCR", Jikai Gene) was added to the control cells.

2.10. Exogenous Gab3 over-expression. The wild-type Gab3 cDNA, synthesized and sequenced by Jikai gene (Shanghai, China), was inserted into p-Super vector (a gift from Dr. Lu [15,16]), which contained a puromycin resistance gene and a Flag tag. The Gab3 construct $(0.25 \mu \mathrm{g} / \mathrm{mL})$ was transfected to CRC cells by Lipofectamine 2000 reagents (Invitrogen, Shanghai, China). Stable cells were again selected via the above puromycin method. Expression of exogenous Gab3 (Flag-tagged) was confirmed by Western blot assay and RT-qPCR assay.

2.11. Tumor xenograft assay. The severe combined immune deficient (SCID) mice (all female, five-week old, 18-19 g weight) were provided by the Animal Center of Fudan University (Shanghai, China). The stable HCT-116 cells, expressing the described shRNA, were s.c. inoculated into the flanks of mice. Palpable tumors started to appear in 3-5 weeks. The size of the tumors was measured weekly by caliper, and tumor volume was calculated: $\pi / 6 \times$ width ${ }^{2} \times$ length [25]. Mice body weights were also recorded weekly. All animal procedures were performed according to the IACUC of all authors' institutions.

2.12. Statistical analysis. The data were presented as means \pm standard deviation (SD). Statistical differences were tested via the one-way ANOVA using the multiple comparisons performed with post hoc Bonferroni test (SPSS 16.0).

\section{Results}




\subsection{Gab3 shRNA knockdown inhibits human CRC cell proliferation}

We fist tested expression of Gab3 in human colon cancer tissue specimens. Western blot assay results in Figure 1A demonstrated that Gab3 protein expression was only seen in the colon cancer tissues ("T"), but was not detected in surrounding normal colon tissues ("N"). qRT-PCR results in Figure 1B confirmed specific Gab3 mRNA expression in cancer tissues, and its level was extremely low in the surrounding normal colon tissues (Figure 1B). Next, we tested expression of Gab3 in human CRC cells. As demonstrated in Figure 1C, Gab3 expression (protein and mRNA) was only detected in established (HCT-116 and HT-29 lines) and primary human CRC cells ("Pri CRC"), but was absent in colon epithelial FHC cells. To test the potential function of Gab3 on CRC cells, shRNA strategy was applied to knockdown Gab3. As described in methods, two non-overlapping lentiviral Gab3 shRNAs (“shGab3-\#1/\#2”) were added to HCT-116 CRC cells. Via puromycin selection, stable cell lines were established. RT-qPCR assay results in Figure 1D demonstrated that the two shRNAs caused significant decrease of Gab3 mRNA in HCT-116 cells. Meanwhile, Gab3 protein expression was also downregulated (Figure 1E). Gab3 expression was unchanged in cells with scramble control shRNA (“sh-SCR") (Figure 1D and E).

Next, we wanted to know if Gab3 knockdown could affect HCT-116 cell proliferation. Various assays including viable cell count assay (Figure 1F), BrdU ELISA assay (Figure 1G) and clonogenic assay (Figure 1H) were employed. Results from these assays showed clearly that HCT-116 cell proliferation was significantly inhibited following Gab3 shRNA knockdown (Figure 1F-H). The viable cell number (at Day-4), BrdU ELISA OD (at Day-4) and number of colonies (at Day-10) were all decreased in cells expressing Gab3 shRNAs (Figure 1F-H). Notably, exact same amount of viable cells were seeded at Day-1 for each assay. Importantly, shGab3-\#2 was more efficient in downregulating Gab3 than shGab3-\#1 (Figure 1D and E), it was also more potent in suppressing cell proliferation (Figure 1F-H). sh-SCR, on the other hand, didn't affect HCT-116 cell proliferation (Figure 1F-H).

The above experiments were also repeated in other CRC cells. In HT-29 cells and primary human colon cancer cells [15], the application of shGab3-\#2 again silenced Gab3 (Figure 1I and J). Similarly, proliferation of above CRC cells, tested by simple viable cell counting assay (at Day-4), was also inhibited (Figure 1K). These results demonstrate that Gab3 shRNA knockdown inhibits CRC cell proliferation. 


\subsection{Gab3 over-expression promotes HCT-116 cell proliferation}

Based upon the above shRNA results, we would speculate that Gab3 over-expression shall promote CRC cell proliferation. Thus, the Gab3 expression vector was constructed (See method), which was transfected to HCT-116 cells. Puromycin was utilized to select stable cells. RT-qPCR assay results in Figure 2A demonstrated that Gab3 mRNA level was significantly increased in stable HCT-116 cells with the construct. Gab3 protein expression was also elevated (Figure 2B). Note that the exogenous Gab3 had a Flag tag (Figure 2B). Remarkably, Gab3 over-expression indeed promoted HCT-116 cell proliferation (Figure 2C-E). The number of viable cells (at Day-4, Figure 2C), the BrdU ELISA OD (at Day-4, Figure 2D) and number of colonies (at Day-10, Figure 2E) were all increased in Gab3-over-expressed cells. As expected, the vector control ("Vector") failed to affect Gab3 expression (Figure $\mathbf{2 A}$ and $\mathbf{B}$ ) and cell proliferation (Figure 2C-E). Thus, these results demonstrate that exogenous over-expression of Gab3 promotes HCT-116 cell proliferation.

\subsection{Gab3 co-precipitates with p85 and SHP2 to mediate Akt and Erk activation in CRC cells}

Gab family proteins are known to dock with p85 and SHP2 to mediate PI3K-Akt and Erk-MAPK activation $[12,13,14]$. Next, co-immunoprecipitation (Co-IP) assay was performed to test such associations. As demonstrated, in response to FBS (10\%, $2 \mathrm{~min})$, Gab3 indeed formed a complex with p85 and SHP2 in HCT-116 cells (Figure 3A) and HT-29 cells (Figure 3B). IgG control failed to induce such associations (Figure 3A and B). Expressions of p85 and SHP2 ("Input") were unchanged in these cells (Figure 3A and B). Remarkably, as shown in Figure 3C, shRNA-mediated knockdown of Gab3 (see Figure 1) inhibited activation of Akt and Erk1/2 (by FBS) in HCT-116 cells and HT-29 cells (Figure 3C). Akt activation was reflected by phosphorylated ("p-") Akt at Thr-308, and Erk activation was evidenced by p-Erk1/2 at Thr-202/Tyr-204 (Figure 3C). Expressions of total Akt1 and Erk1/2 were unchanged (Figure 3C). On the other hand, exogenous over-expression of Gab3 (see Figure 2) augmented Akt and Erk activation in HCT-116 cells (Figure 3D). These results suggest that Gab3 coprecipitates with p85 and SHP2 to mediate Akt and Erk activation in CRC cells.

\subsection{In vivo growth of HCT-116 tumors in mice is suppressed following expressing Gab3 shRNA}

At last, SCID mice tumor xenograft model was applied to study the potential function of Gab3 on CRC cells in vivo. Same amount of HCT-116 cells (eight million cells per mouse), expressing nonsense scramble shRNA ("sh-SCR") or Gab3 shRNA ("shGab3-\#1/\#2", see Figure 1) were s.c. injected 
to the SCID mice. Recording was started when tumors reached volumes around $100 \mathrm{~mm}^{3}$ (for each group). As demonstrated, HCT-116 tumors with Gab3 shRNA (-\#1/\#2) were much smaller than those expressing control shRNA (Figure 4A). Further, Gab3 shRNA-expressing tumors grew significantly slower than the control tumors (Figure 4B). The weighs of tumors with Gab3 shRNA (at Wk-7) were also significantly lighter $v s$. control tumors (Figure 4C). Mice body weights were indifferent between the groups (Figure 4D). Tumor tissues were then isolated and subjected to signaling assessment. As shown in Figure 4E and F, Gab3 expression was indeed depleted in tumors with Gab3 shRNA. Significantly, Akt and Erk activation was also largely inhibited in the above tumors (Figure $\mathbf{4 E}$ and $\mathbf{F}$ ). Therefore, in line with the in vitro findings, Gab3 shRNA knockdown also inhibited Akt and Erk activation in HCT116 tumors (Figure 4E and F). Notably, shGab3-\#2 was again more efficient in downregulating Gab3 than shGab3-\#1 in vivo (Figure 4E and F). It was also more dramatic in suppressing tumor growth (Figure $\mathbf{4 A - C}$ ) and Akt-Erk activation (Figure $\mathbf{4 E}$ and $\mathbf{F}$ ).

\section{Discussions}

Gab family adaptor proteins, including Gab1/2/3, are known to dock with multiple signaling proteins, including p85, PLCY, SHP2, sarcoma ct10 oncogene homolog (Crk) and casitas B-lineage lymphoma proto-oncogene (c-Cbl) [11,26,27]. This will mediate activation of multiple downstream signalings, i.e. Akt-mTOR, Erk-MAPK and p38 cascades [11,26,27]. Activation of these oncogenic signalings is important for CRC cell progression [1,8,9]. Recent studies have focused on expression and potential functions of Gab1, the most dominant Gab family protein, in CRC [10,28,29]. It has been shown that Gab1 is over-expressed and/or over-activated in CRC, which is associated with cancer cell progression $[10,28,29]$. For example, An et al., showed that miR-5582-5p selectively degrades Gab1 to inhibit CRC cells [29]. Similarly, Bai et al., showed that microRNA-409-3p inhibits CRC cell invasion and metastasis possibly via depleting Gab1 [28]. Further, HGF-mediated in colon cancer cell differentiation also requires Gab1 [30]. Gab3, on the other hand, is the least ubiquitously expressed member of the Gab family protein, which is mainly expressed in hematopoietic cells/tissues $[12,13,14]$. To our best knowledge, this is the first report studying Gab3 expression and its biological functions in CRC cells.

The results of this study suggest that Gab3 might be an important oncogene of CRC. First, Gab3 is only expressed in human colon cancer tissues and CRC cells, but not in normal human colon tissues and colon epithelial cells. Second, shRNA-mediated Gab3 knockdown inhibited CRC cell proliferation. On the other hand, exogenous over-expression of Gab3 promoted HCT-116 cell 
proliferation. Significantly, Gab3 was shown to co-precipitate with p85 and SHP2 in CRC cells, which apparently was important for subsequent Akt and Erk activation. Gab3 shRNA knockdown inhibited Akt and Erk activation in CRC cells, while exogenous Gab3 over-expression augmented it. In vivo, the growth of HCT-116 tumors in SCID mice was also inhibited following expressing Gab3 shRNA. Meanwhile, Akt and Erk activation was also significantly inhibited in Gab3 shRNA-expressing tumors. Together, the results of this preclinical study suggest that Gab3 might be an important oncogene or oncotarget protein of CRC. Future studies will be needed to further test expression of this Gab protein in CRC tissues. The underlying mechanisms of Gab3-mediated Akt-Erk activation warrant further investigations.

5. Acknowledgments. This work is supported by the National Science Foundation of China.

\section{References}

[1] H.J. Schmoll, A. Stein, Colorectal cancer in 2013: Towards improved drugs, combinations and patient selection, Nat Rev Clin Oncol 11 (2014) 79-80.

[2] E.J. Kuipers, T. Rosch, M. Bretthauer, Colorectal cancer screening--optimizing current strategies and new directions, Nat Rev Clin Oncol 10 (2013) 130-142.

[3] A. Brouquet, B. Nordlinger, Metastatic colorectal cancer outcome and fatty liver disease, Nat Rev Gastroenterol Hepatol 10 (2013) 266-267.

[4] R. Siegel, J. Ma, Z. Zou, A. Jemal, Cancer statistics, 2014, CA Cancer J Clin 64 (2014) 9-29.

[5] W. Chen, R. Zheng, P.D. Baade, S. Zhang, H. Zeng, F. Bray, A. Jemal, X.Q. Yu, J. He, Cancer statistics in China, 2015, CA Cancer J Clin (2016).

[6] W. Chen, Cancer statistics: updated cancer burden in China, Chin J Cancer Res 27 (2015) 1.

[7] J.M. Hubbard, A. Grothey, Colorectal cancer in 2014: Progress in defining first-line and maintenance therapies, Nat Rev Clin Oncol 12 (2015) 73-74.

[8] I. Leake, Colorectal cancer: Understanding the routes of metastasis in colorectal cancer, Nat Rev Gastroenterol Hepatol 11 (2014) 270.

[9] D. Kerr, Clinical development of gene therapy for colorectal cancer, Nat Rev Cancer 3 (2003) 615-622.

[10] A. Yart, P. Mayeux, P. Raynal, Gab1, SHP-2 and other novel regulators of Ras: targets for anticancer drug discovery?, Curr Cancer Drug Targets 3 (2003) 177-192.

[11] H. Gu, B.G. Neel, The "Gab" in signal transduction, Trends Cell Biol 13 (2003) 122-130.

[12] M. Seiffert, J.M. Custodio, I. Wolf, M. Harkey, Y. Liu, J.N. Blattman, P.D. Greenberg, L.R. Rohrschneider, Gab3-deficient mice exhibit normal development and hematopoiesis and are immunocompetent, Mol Cell Biol 23 (2003) 2415-2424.

[13] C. Bourgin, R.P. Bourette, S. Arnaud, Y. Liu, L.R. Rohrschneider, G. Mouchiroud, Induced expression and association of the Mona/Gads adapter and Gab3 scaffolding protein during monocyte/macrophage differentiation, Mol Cell Biol 22 (2002) 3744-3756.

[14] I. Wolf, B.J. Jenkins, Y. Liu, M. Seiffert, J.M. Custodio, P. Young, L.R. Rohrschneider, Gab3, a new DOS/Gab family member, facilitates macrophage differentiation, Mol Cell Biol 22 (2002) 231-244.

[15] P.H. Lu, M.B. Chen, C. Ji, W.T. Li, M.X. Wei, M.H. Wu, Aqueous Oldenlandia diffusa 
extracts inhibits colorectal cancer cells via activating AMP-activated protein kinase signalings, Oncotarget (2016).

[16] M.B. Chen, L. Yang, P.H. Lu, X.L. Fu, Y. Zhang, Y.Q. Zhu, Y. Tian, MicroRNA-101 downregulates sphingosine kinase 1 in colorectal cancer cells, Biochem Biophys Res Commun 463 (2015) 954-960.

[17] C. Li, J.F. Cui, M.B. Chen, C.Y. Liu, F. Liu, Q.D. Zhang, J. Zou, P.H. Lu, The preclinical evaluation of the dual $\mathrm{mTORC1/2}$ inhibitor INK-128 as a potential anti-colorectal cancer agent, Cancer Biol Ther 16 (2015) 34-42.

[18] M.B. Chen, X.Y. Wu, G.Q. Tao, C.Y. Liu, J. Chen, L.Q. Wang, P.H. Lu, Perifosine sensitizes curcumin-induced anti-colorectal cancer effects by targeting multiple signaling pathways both in vivo and in vitro, Int J Cancer 131 (2012) 2487-2498.

[19] M.B. Chen, M.X. Wei, J.Y. Han, X.Y. Wu, C. Li, J. Wang, W. Shen, P.H. Lu, MicroRNA-451 regulates AMPK/mTORC1 signaling and fascin1 expression in HT-29 colorectal cancer, Cell Signal 26 (2014) 102-109.

[20] T. Yu, J. Ji, Y.L. Guo, MST1 activation by curcumin mediates JNK activation, Foxo3a nuclear translocation and apoptosis in melanoma cells, Biochem Biophys Res Commun 441 (2013) 53-58.

[21] C. Cao, X. Huang, Y. Han, Y. Wan, L. Birnbaumer, G.S. Feng, J. Marshall, M. Jiang, W.M. Chu, Galpha(i1) and Galpha(i3) are required for epidermal growth factor-mediated activation of the Akt-mTORC1 pathway, Sci Signal 2 (2009) ra17.

[22] W. Shioyama, Y. Nakaoka, K. Higuchi, T. Minami, Y. Taniyama, K. Nishida, H. Kidoya, T. Sonobe, H. Naito, Y. Arita, T. Hashimoto, T. Kuroda, Y. Fujio, M. Shirai, N. Takakura, R. Morishita, K. Yamauchi-Takihara, T. Kodama, T. Hirano, N. Mochizuki, I. Komuro, Docking protein Gab1 is an essential component of postnatal angiogenesis after ischemia via HGF/c-met signaling, Circ Res 108 (2011) 664-675.

[23] Y. Lu, J. Gao, Down-expression pattern of Ku70 and p53 coexisted in colorectal cancer, Med Oncol 32 (2015) 98.

[24] Y. Lu, J. Gao, Downregulated Ku70 and ATM associated to poor prognosis in colorectal cancer among Chinese patients, Onco Targets Ther 7 (2014) 1955-1961.

[25] L.S. Qin, P.F. Jia, Z.Q. Zhang, S.M. Zhang, ROS-p53-cyclophilin-D signaling mediates salinomycin-induced glioma cell necrosis, J Exp Clin Cancer Res 34 (2015) 57.

[26] Y. Liu, L.R. Rohrschneider, The gift of Gab, FEBS Lett 515 (2002) 1-7.

[27] M. Hibi, T. Hirano, Gab-family adapter molecules in signal transduction of cytokine and growth factor receptors, and $T$ and $B$ cell antigen receptors, Leuk Lymphoma 37 (2000) 299-307.

[28] R. Bai, C. Weng, H. Dong, S. Li, G. Chen, Z. Xu, MicroRNA-409-3p suppresses colorectal cancer invasion and metastasis partly by targeting GAB1 expression, Int J Cancer 137 (2015) 2310-2322.

[29] H.J. An, S.Y. Kwak, J.O. Yoo, J.S. Kim, I.H. Bae, M.J. Park, M.Y. Cho, J. Kim, Y.H. Han, Novel miR-5582-5p functions as a tumor suppressor by inducing apoptosis and cell cycle arrest in cancer cells through direct targeting of GAB1, SHC1, and CDK2, Biochim Biophys Acta 1862 (2016) 1926-1937.

[30] S. Kermorgant, V. Dessirier, M.J. Lewin, T. Lehy, HGF upregulates and modifies subcellular distribution of proteins in colon cancer cell enterocytic differentiation, Am J Physiol Gastrointest Liver Physiol 281 (2001) G1068-1080.

8. Figure legends.

Figure 1. Gab3 shRNA knockdown inhibits human CRC cell proliferation. Human colon 
cancer tissues ("T", $n=10$ ) and paired surrounding normal colon tissues ("N", n=10) were lysed in tissue lysis buffer, Gab3 and $\beta$-actin (equal loading) expressions were tested by Western blot assay (A, representative blots of four paired tissues were shown) and qRT-PCR (B) assay. Expression of Gab3 in listed cells was tested by Western blot assay and RT-qPCR assay (C). Stable HCT-116 cells, HT-29 cells or primary human colon cancer cells ("Pri CRC”), with Gab3 shRNA (“shGab3-\#1/\#2”) or nonsense scramble shRNA ("sh-SCR"), were subjected to RT-qPCR assay (D and I) and Western blot assay ( $\mathbf{E}$ and $\mathbf{J}$ ) to test Gab3 expression; Cell proliferation was tested by designated assays (F-H and K). "Parental" stands for parental control cells (Same for all Figures). Bars stand for mean \pm SD (Same for all figures). Gab3 protein expression was normalized to $\beta$-actin $(\mathbf{A}, \mathbf{C}, \mathbf{E}$ and $\mathbf{J})$. Experiments in this figure were repeated four times, with similar results obtained. * $\boldsymbol{p}<0.05$ vs. "N", "Parental" or "sh-SCR" group.

Figure 2. Gab3 over-expression promotes HCT-116 cell proliferation. Stable HCT-116 cells, expressing wild-type Gab3 cDNA(“wt-Gab3”) or empty vector ("Vector", p-Super), were subjected to RT-qPCR assay (A) and Western blot assay (B) of Gab3 expression; Note that the exogenous Gab3 was tagged with Flag (B). Cell proliferation was tested by described assays (C-E). Relative Gab3 protein expression was normalized to $\beta$-actin $(\mathbf{B})$. Experiments in this figure were repeated four times, with similar results obtained. * $\boldsymbol{p}<0.05$ vs. "Parental" cells.

Figure 3. Gab3 co-precipitates with $\mathrm{p85}$ and SHP2 to mediate Akt and Erk activation in CRC cells. HCT-116 cells (A) and HT-29 cells (B) were PBS starved ("PBS", $30 \mathrm{~min}$ ) or stimulated with FBS (10\%, 2 min), the association between Gab3 and p85 or SHP2 was detected by coimmunoprecipitation (“Co-IP”) assay, expressions of above proteins were also tested by Western blot assay as "Inputs". HCT-116 cells or HT-29 cells, expressing Gab3 shRNA(“shGab3-\#2”) or non-sense scramble shRNA ("sh-SCR"), were subjected to Western blot assay of listed proteins (C). HCT-116 cells expressing wild-type Gab3 cDNA ("wt-Gab3") or empty vector ("Vector", p-Super) were also subjected to Western blot assay (D). Akt phosphorylation (vs. total Akt1) and Erk1/2 phosphorylation (vs. total Erk1/2) were quantified (C and D, lower panels). Experiments in this figure were repeated four times, with similar results obtained. * $\boldsymbol{p}<0.05$ vs. "sh-SCR" group (C) or "Vector" group (D).

Figure 4. In vivo growth of HCT-116 tumors in mice is suppressed following expressing Gab3 shRNA. SCID mice (n=8 per group), bearing HCT-116 tumors with Gab3 shRNA(“shGab3-\#1/\#2”) or non-sense scramble shRNA (“sh-SCR”), were monitored: Tumor volumes (A) and mice body weights (D) were measured weekly; Estimated daily tumor growth was calculated (B); Tumor weights at the 
end of experiments (Week-7) were also recorded (C). At Week-4 and Week-7, one tumor per group was isolated, expressions of listed proteins in tumor lysates were tested ( $\mathbf{E}$ and $\mathbf{F})$. Gab3 expression (vs. $\beta$-actin), Akt phosphorylation ( $v s$. total Akt1) and Erk1/2 phosphorylation (vs. total Erk1/2) were quantified (E and F). "Wk" stands for Week. * $\boldsymbol{p}<0.05$ vs. "sh-SCR" group. ${ }^{\#} \boldsymbol{p}<0.05$ vs. "shGab3\#1” group. 
A.
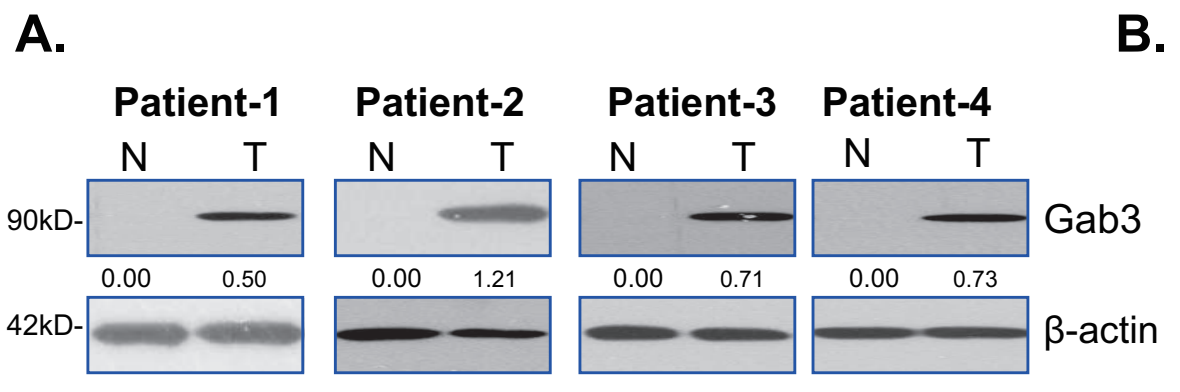

C.

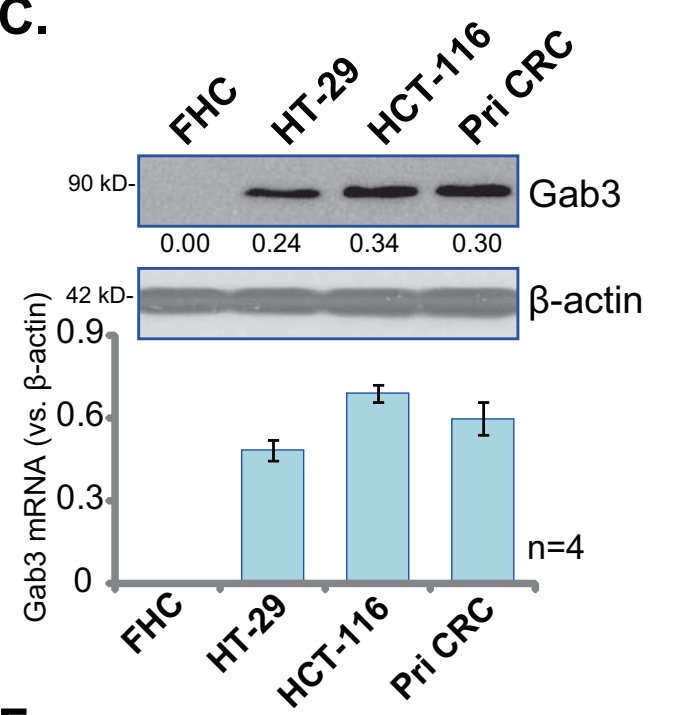

$\mathbf{F}$.

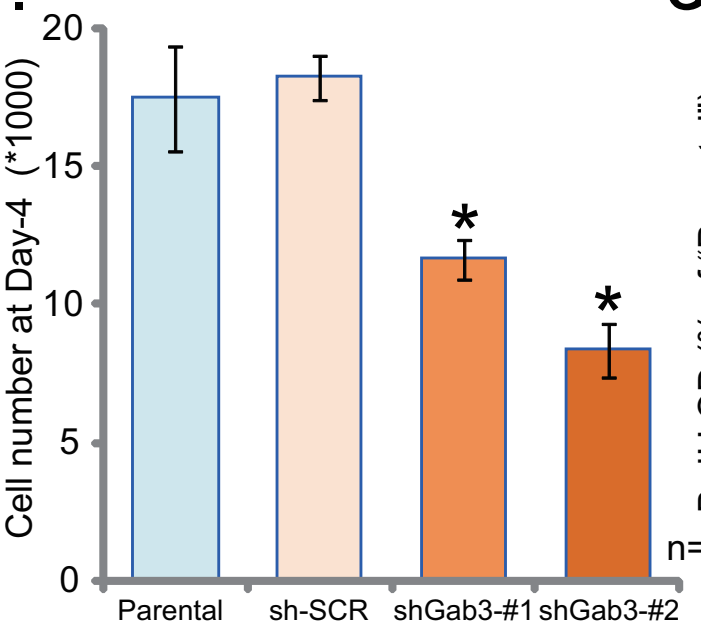

I.

.

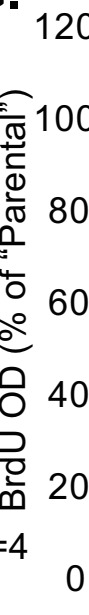

J.
D.

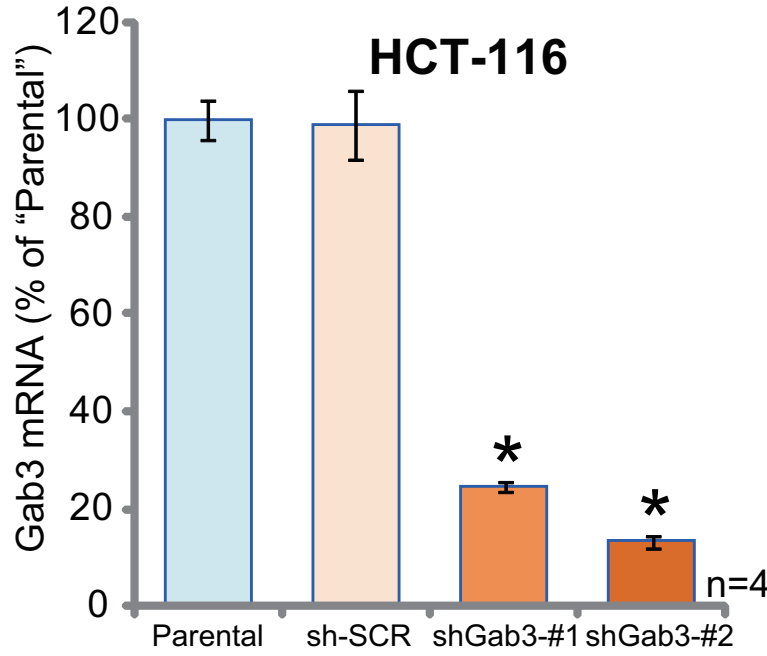

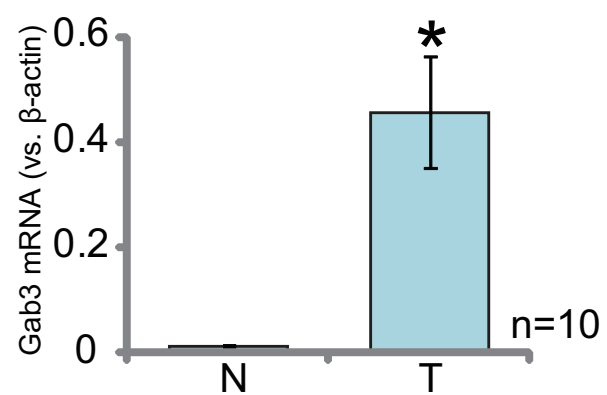

E.

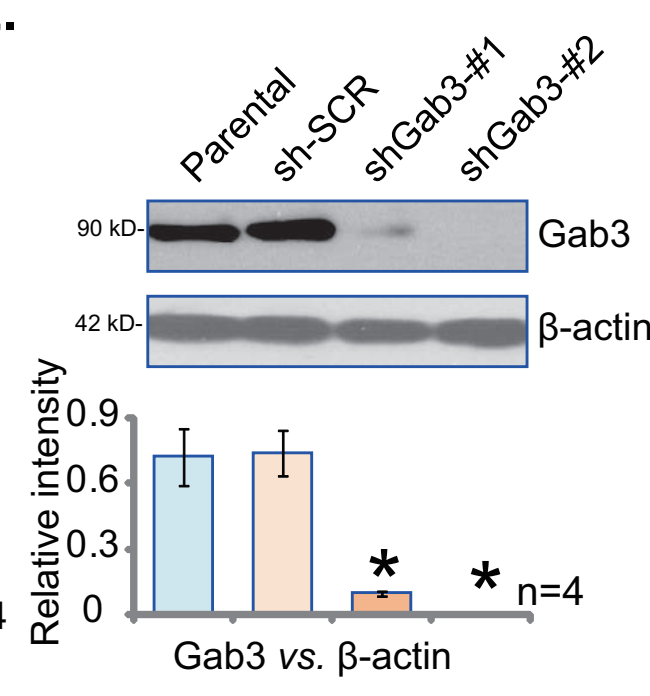

H.

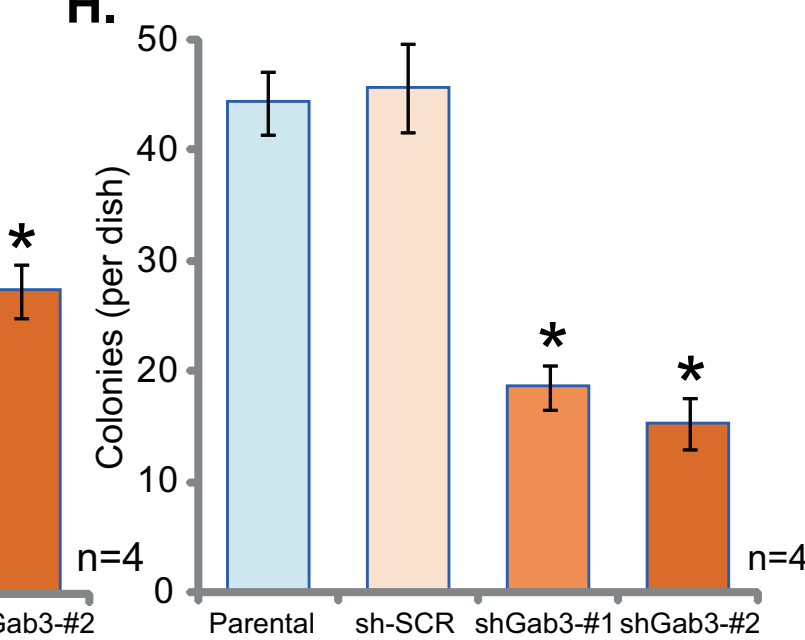

$\mathrm{K}$.

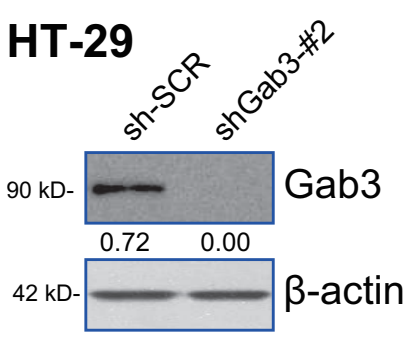

Pri CRC

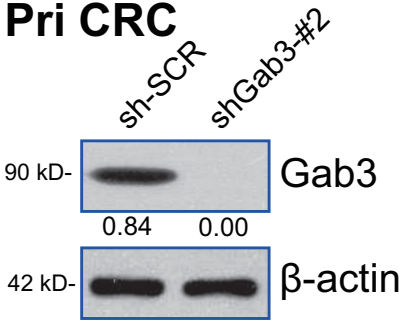

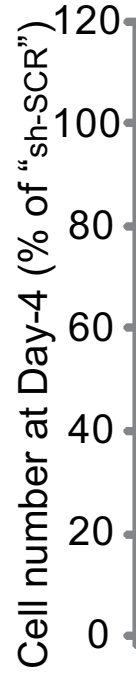

sh-SCR shGab3-\#2 sh-SCR shGab3-\#2 
Figure 2.

A.

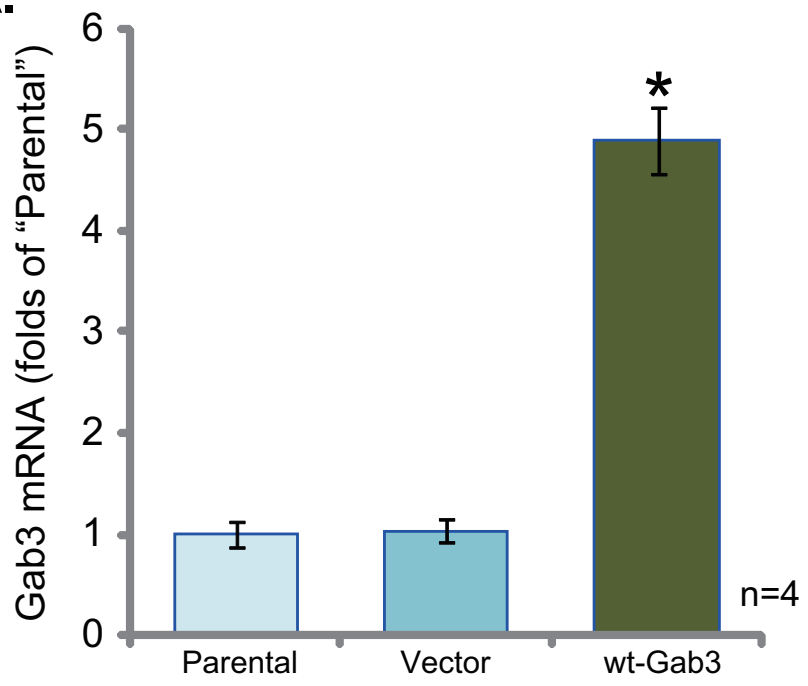

C.

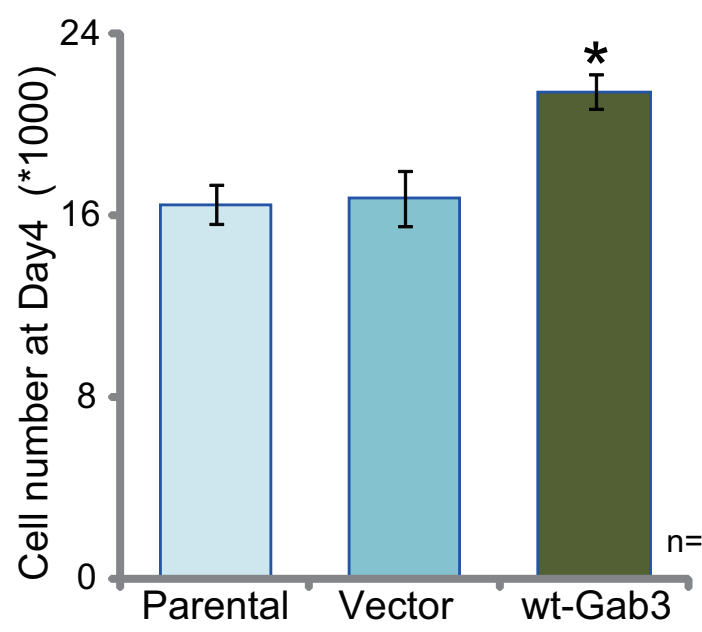

B.

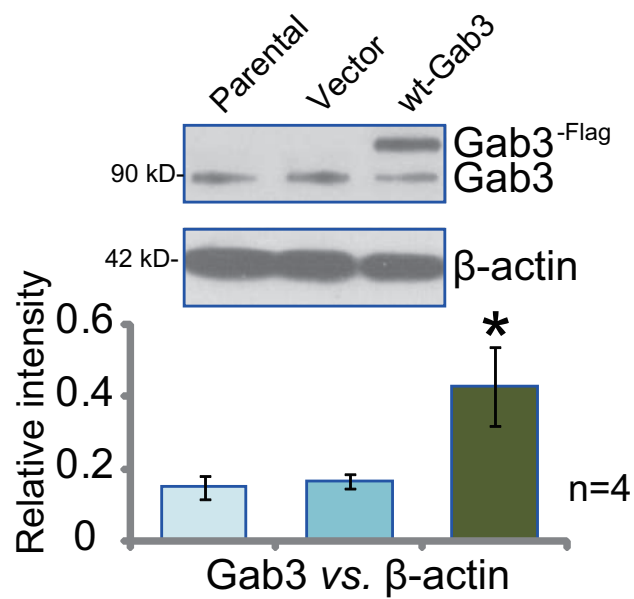

E.

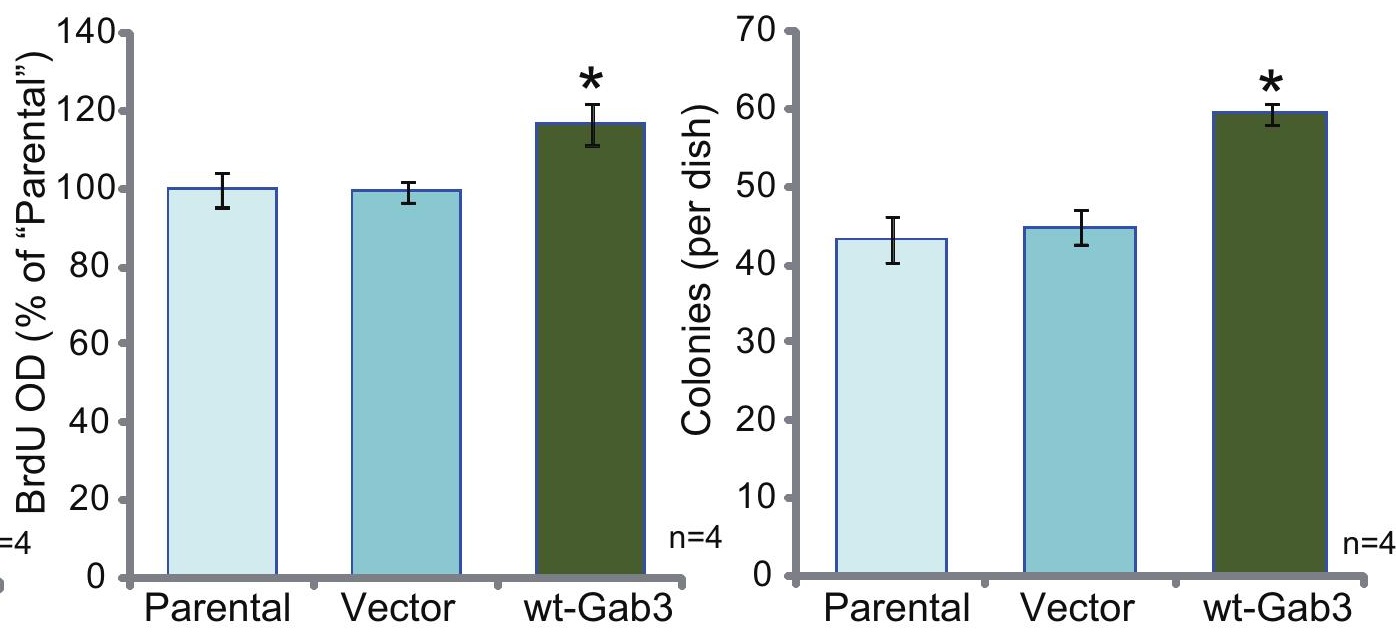


A.

HCT-116

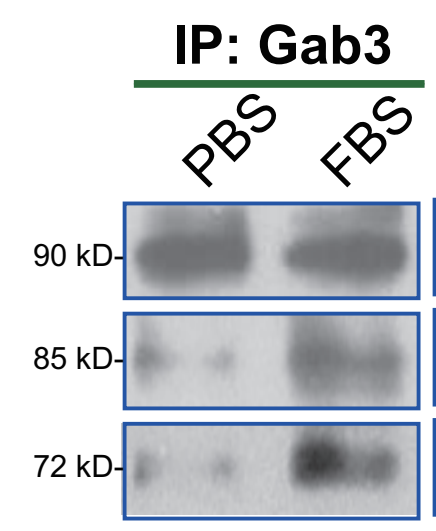

\section{Inputs:}

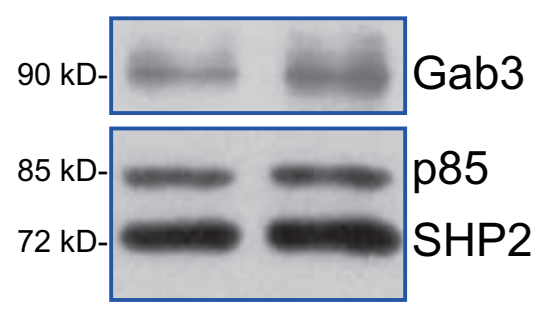

C.

\section{HCT-116 HT-29}
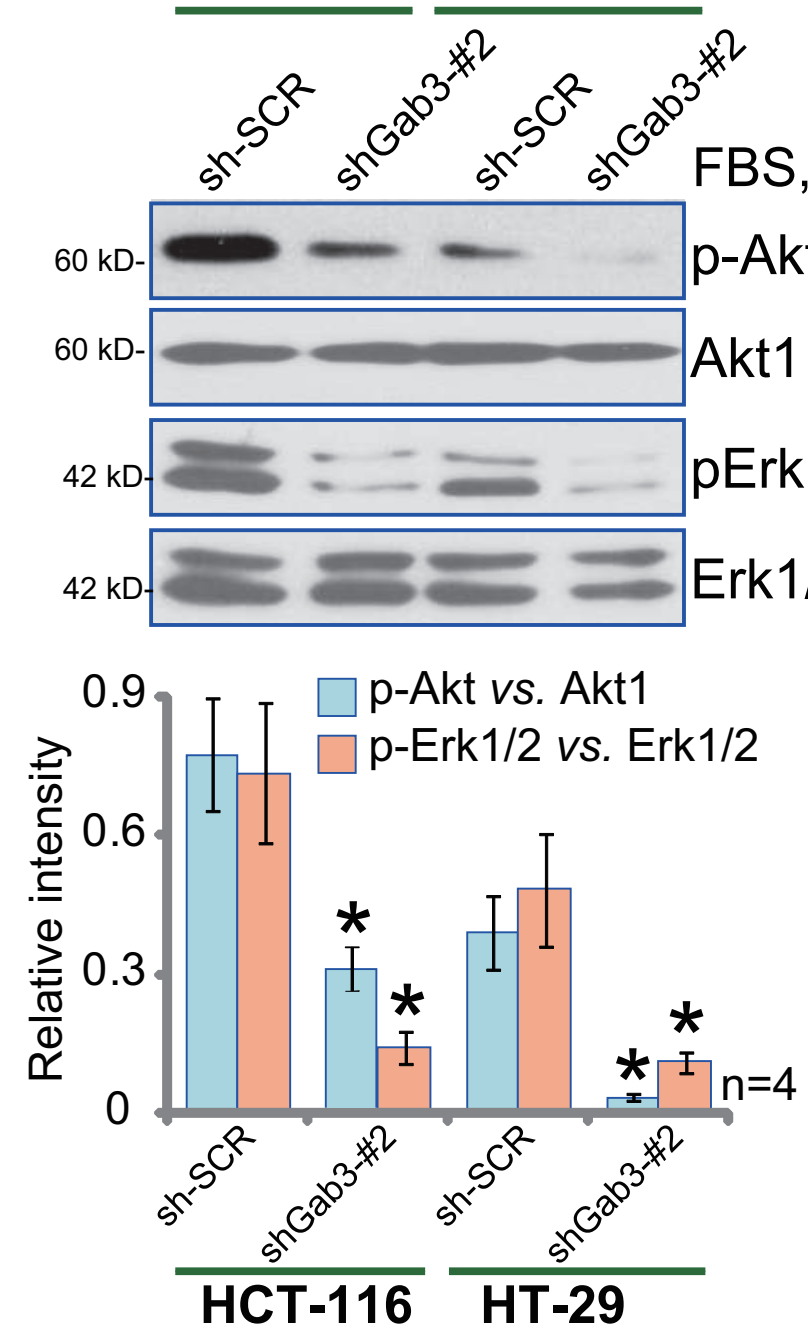

\section{HT-29}
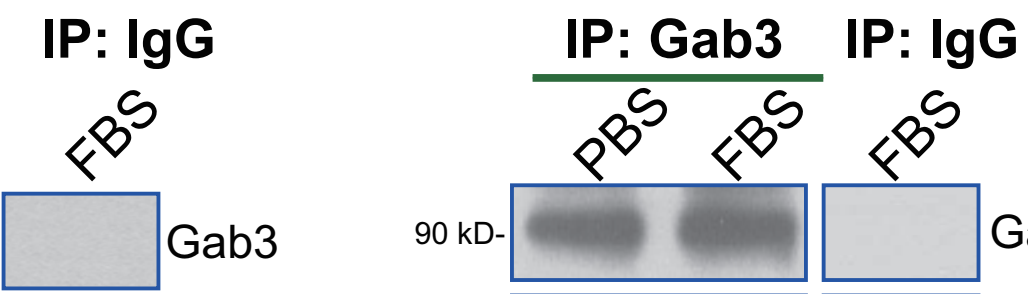

SHP2

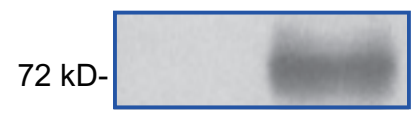

\section{Inputs:}

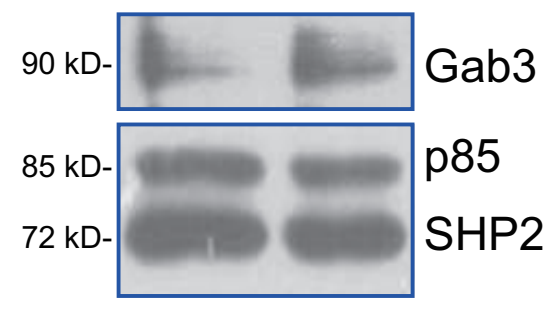

D.

\section{HCT-116}

FBS, 5'

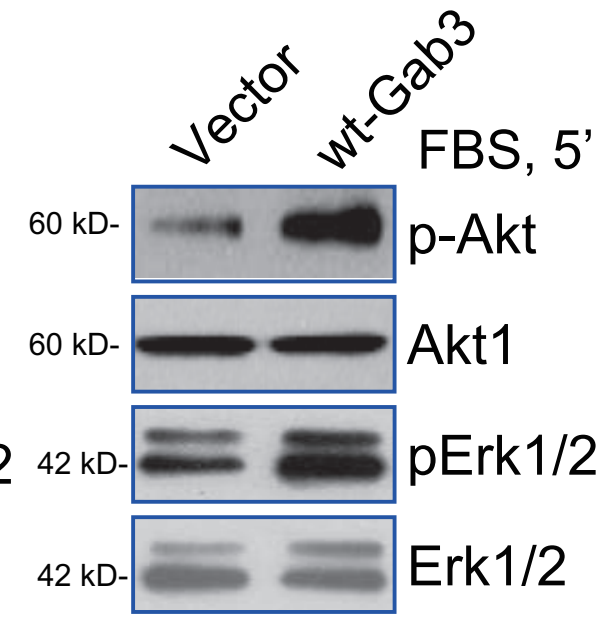

\section{Akt1}

pErk1/2

Erk1/2

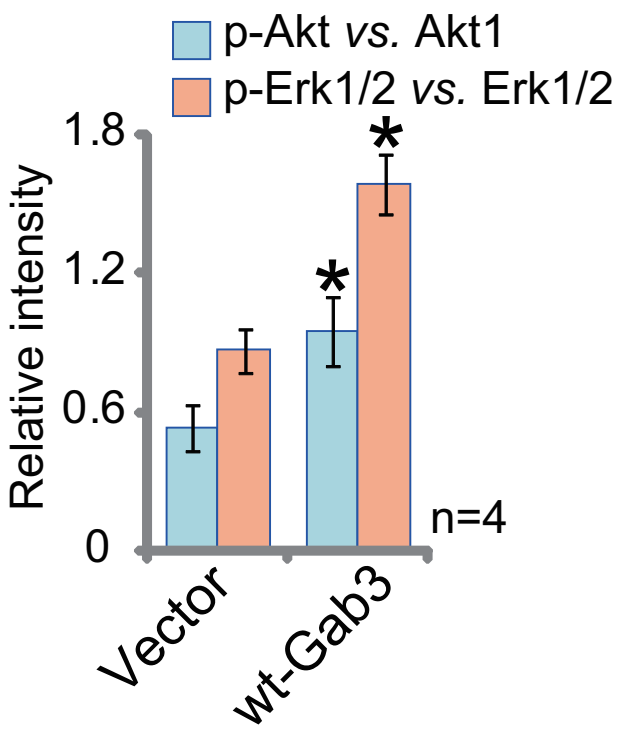


Figure 4.

A.

A.

$A$
4
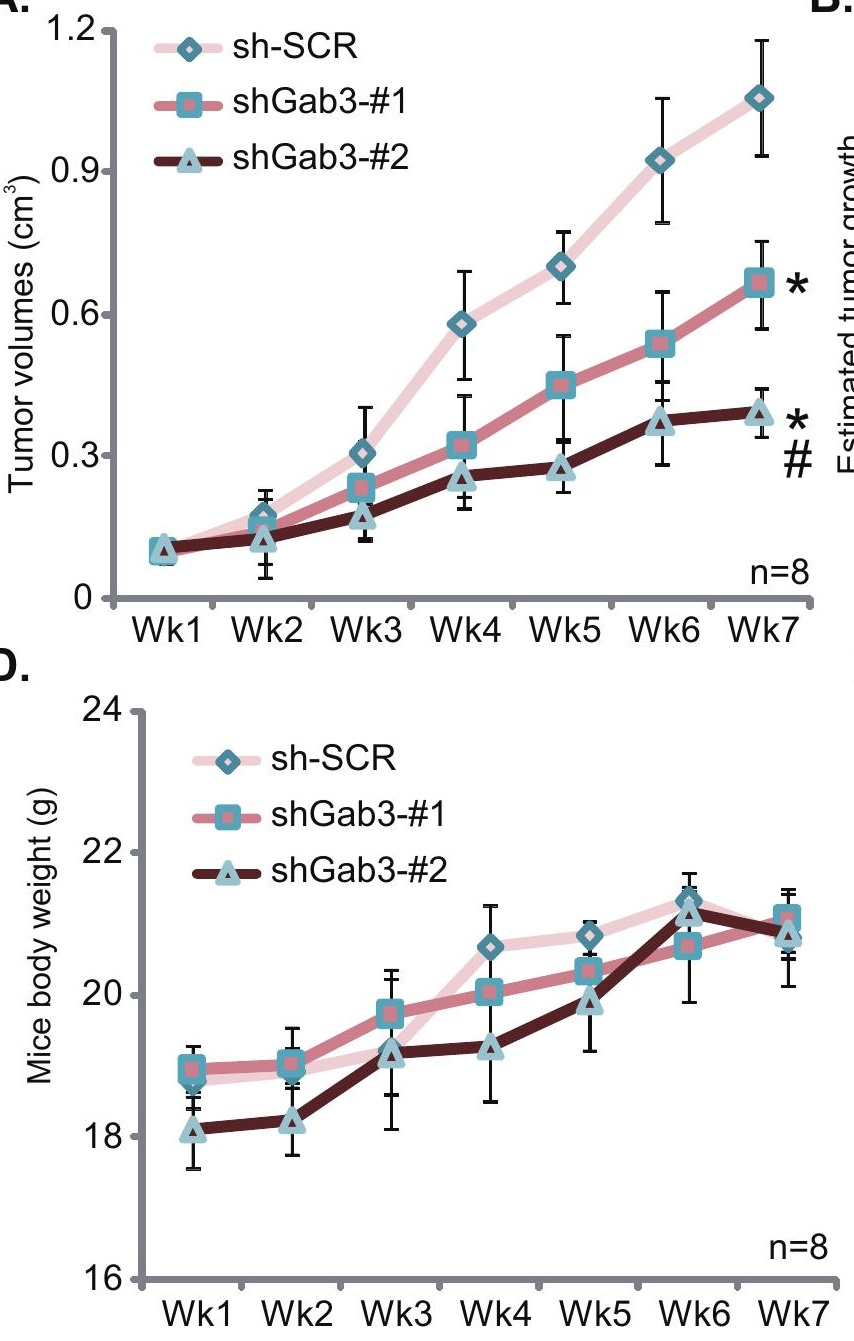

B.

D. Wk1 Wk2 Wk3 Wk4 Wk5 Wk6 Wk7
E.

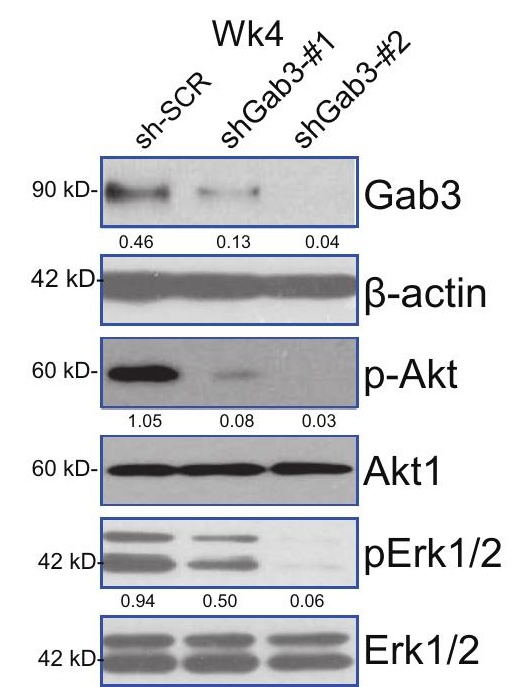

C.
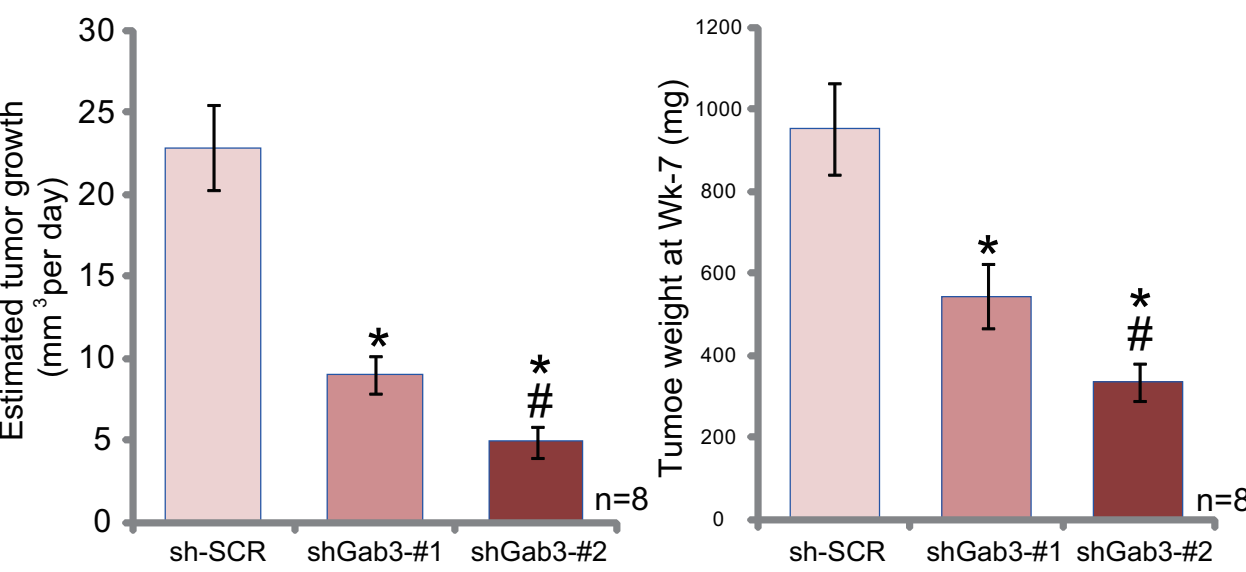

F.

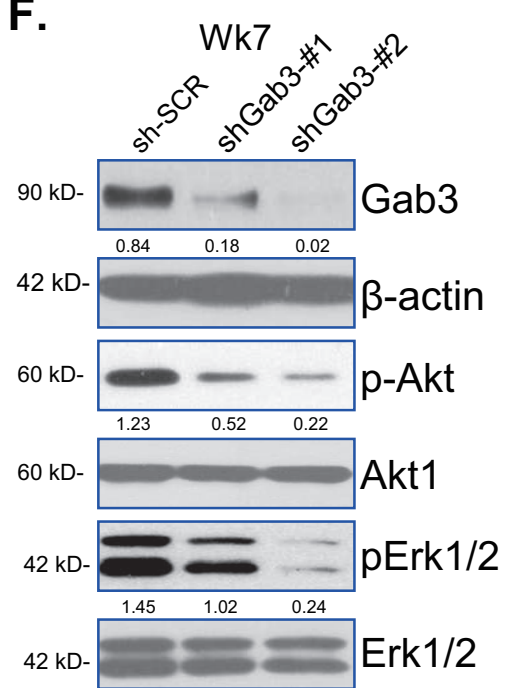

